consequences has far reaching implications for how we care for this vulnerable group especially when it comes to nursing them on ward areas rather than the HDU setting. The findings from this study have proved fascinating and set the scene for further exploration.

\section{P155 NON-INVASIVE VENTILATION (NIV) IN ACUTE HYPERCAPNIC RESPIRATORY FAILURE IN RESTRICTIVE LUNG DISEASES (RLD)}

doi:10.1136/thx.2010.151043.6

${ }^{1} \mathrm{~A}$ Thomas, ${ }^{1} \mathrm{~A}$ Ali, ${ }^{2} \mathrm{~B}$ Chakraborty, ${ }^{1} \mathrm{~B}$ Beauchamp, ${ }^{1} \mathrm{E}$ Gallagher, ${ }^{1} \mathrm{D}$ Banerjee, ${ }^{1} \mathrm{R}$ Mukherjee. ${ }^{1}$ Heart of England NHS Foundation Trust, Birmingham, UK; ${ }^{2}$ School of Mathematics, University of Birmingham, Birmingham, UK

Introduction Successful NIV has been described in RLD (thoracic cage disease, obesity-hypoventilation and neuromuscular diseases) and given the success in chronic ventilatory failure, NIV should be considered as the treatment of choice in decompensated ventilatory failure due to RLD in an acute setting.

Methods Analysis of initial ABG in those admitted to a NIV unit on a respiratory ward with a diagnosis of RLD, requiring NIV for an acute episode of respiratory failure $\left(\mathrm{pH}<7.35\right.$ and $\left.\mathrm{pCO}_{2}>6.0 \mathrm{kPa}\right)$ admitted between 01 August 2004 and 31 December 2009. Patients included were those with respiratory failure as a consequence of RLD. Those who were either admitted or managed in HDU were excluded from the analysis. The admission episodes were stratified by initial $\mathrm{pH}$ ranges (predictor variable) and in-hospital mortality was recorded (outcome variable).

Results In 270 admissions (221 unique patients) with RLD requiring NIV for acute respiratory failure, the overall mortality was 35 (13.0\%). There was a significant increase in mortality in the group where ABG $\mathrm{pH}<7.15$ (40.9\%). The difference is significant when compared to the group with a $\mathrm{pH}$ between 7.15 and $7.26(9.6 \%)(\mathrm{p}=0.027)$. There was no significant difference in mortality in the group with $\mathrm{pH} 7.15$ to 7.26 to the group with $\mathrm{pH} 7.26$ to 7.35 ( $\mathrm{p}=0.94$ ): See Abstract P155 Figure 1 . The plot of fitted logistic regression equation with initial $\mathrm{pH}$ (the dotted lines indicate $95 \%$ confidence band).

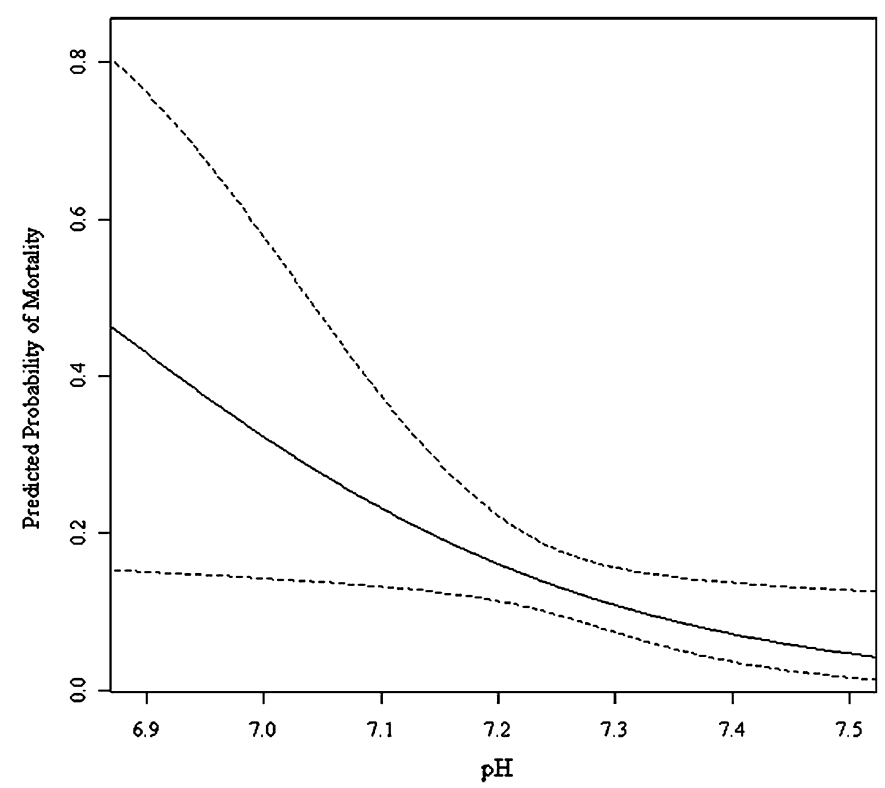

Abstract P155 Figure 1 The plot of fitted logistic regression equation with initial $\mathrm{pH}$ (the dotted lines indicate 95\% confidence band).

Conclusion For those patients with RLD treated with NIV on a respiratory ward, the mortality increases with the severity of acidosis. As no current guidelines indicate a $\mathrm{pH}$ cut-off for the ward based management of RLD with NIV, from our results we propose that this could be an initial ABG pH of 7.15.

\section{P156 DOES ANALYSIS OF PATIENT-VENTILATOR INTERACTION OFFER BENEFITS IN ADDITION TO OVERNIGHT PULSE OXIMETRY IN PATIENTS WITH MOTOR NEURONE DISEASE BEING FOLLOWED ON NON-INVASIVE VENTILATION?}

doi:10.1136/thx.2010.151043.7

${ }^{1} \mathrm{~B}$ Chakrabarti, ${ }^{2} \mathrm{H}$ Ando, ${ }^{3} \mathrm{E}$ Thornton, ${ }^{4} \mathrm{~V}$ Ford, ${ }^{2} \mathrm{C}$ A Young, ${ }^{1} \mathrm{R} \mathrm{M}$ Angus. ${ }^{1}$ Aintree Chest Centre, University Hospital Aintree, Liverpool, UK; ${ }^{2}$ Walton Centre for Neurology and Neurosurgery, Liverpool, UK; ${ }^{3}$ School of Psychology, University of Liverpool, Liverpool, UK; ${ }^{4}$ Department of Physiotherapy, University Hospital Aintree, Liverpool, UK

Introduction Non-invasiveventilation (NIV) is increasingly being offered to Motor Neurone Disease (MND) patients as studies report benefit insurvival and quality of life (QoL). Ventilator technology allows monitoring of patient-ventilator interaction. It is less clear whether such data yield useful additional information over pulse overnight oximetry. In a longitudinal study, MND patients were assessed both physiologically and psychologically prior to NIV initiation and 3monthly until death.

Methods 35 patients were enrolled; 28 offered NIV; 11 declined and 17 established on treatment. At initiation all had nocturnal hypoventilation by symptoms andoximetry criteria. Nine patients ( 8 male; median age 60 years) had $\geq 12$ months follow-up on ventilation; none required oxygen with NIV. Patient-ventilator interaction was assessed by analysis of the 'memory card' from the ventilator at 4-6 months post initiation (Point A) and at 10-12 months (Point B); QoL assessed by validated questionnaires within 2 weeks of memory card analysis.

Results Median overnight oxygen saturation in the sample was $93 \%$ at point $\mathrm{A}$ and $94 \%$ at $\mathrm{B}$; only 3 and 2 patients respectively exhibited 'sub-optimal' oximetry that is, >30 min below $90 \%$ saturation resulting in adjustment of ventilation. Minute Ventilation (MV) fell in the group from Point A to B (mean 6.97-6.34 1/min); a fall in MV (noted in five patients of which 1 exhibited "sub-optimal" oximetry) was associated with a fall in ALS FRS score denoting worsening health status (correlation coefficient $0.73 ; p=0.026$ ). Ventilator triggering decreased overall in the group from Points A to B (mean 69.95-61.57\% proportion of triggered breaths); a decrease in ventilator triggering (noted in six patients of which 1 exhibited "suboptimal" oximetry) correlated with an increase in ALS AQ domain for emotion denoting worsening emotional functioning (correlation coefficient -0.89; $\mathrm{p}=0.002$ ) with anon-significant trend noted between fall in triggered breaths and increase in Hospital Anxiety and Depression (HAD) score (correlation coefficient -0.68 ; $\mathrm{p}=0.055$ ).

Conclusion In MND, monitoring of patient-ventilator interaction may serve as a useful adjunct to pulse oximetry and symptom assessment. Further studies are needed to ascertain whether adjustment of ventilation based on this approach allows patients to gain full benefit from NIV.

\section{P157 STAFF EDUCATION IMPROVES NON-INVASIVE VENTILATION OUTCOMES IN COPD PATIENTS PRESENTING WITH ACUTE HYPERCAPNOEIC RESPIRATORY FAILURE.}

doi:10.1136/thx.2010.151043.8

S Roychowdhury, O Kankam, C Jackson, D Sword, K Prasad, V Stark. The Ayr Hospital, NHS Ayrshire \& Arran, Ayr, UK

Introduction Non invasive ventilation (NIV) is safe and effective treatment for acute hypercapnoeic respiratory failure. National 